\title{
Pregnancy Following Hysteroscopic Resection of a Leiomyoma and Oocyte Donation
}

\author{
MARK V. SAUER, M.D., and CHARLES M. MARCH, M.D.
}

\begin{abstract}
A successful pregnancy was established in a functionally agonadal woman using oocyte donation following resection of a large leiomyoma. The patient had a $6 \times 6 \mathrm{~cm}$ intramural tumor with intracavitary extension. She was symptomatic with metrorrhagia. A conservative approach was undertaken in an attempt to restore normal endometrial anatomy. Hysteroscopic resection removed a $3 \times 3 \times 2 \mathrm{~cm}$ mass. Postoperatively, hysteroscopy was normal, and oocyte donation was performed. Implantation occurred, and a living, $3600 \mathrm{~g}$ female infant was delivered vaginally at 40 weeks gestation without complication. This case illustrates an approach to managing cavity-distorting leiomyomas in perimenopausal women interested in oocyte donation. When hysteroscopic resection of leiomyomas is successful, major surgery is avoided, and pregnancy, labor, and delivery may proceed normally. (J GYNECOL SURG 10:255, 1994)
\end{abstract}

\section{INTRODUCTION}

$\mathbf{O}$ OCYTE DONATION HAS BECOME the treatment of choice for infertile perimenopausal women who are attempting pregnancy. ${ }^{1-3}$ Patients with elevated serum gonadotropins have limited success using any of the other assisted reproductive methods. ${ }^{4}$ Published criteria for accepting patients into an oocyte donation program generally have required demonstration of a normal intrauterine cavity, defined either by hysterosalpingogram (HSG) or hysteroscopy. ${ }^{2,3}$

We have reported good success rates for both embryo implantation and delivery in women undergoing oocyte donation who are known to have asymptomatic leiomyomas without submucosal extension. ${ }^{5}$ In our experience, prior myomectomy has not improved results in terms of obstetric outcome. However, women choosing not to undergo myomectomy have experienced a significantly greater incidence of pelvic pain during the first trimester. We report a patient with a large myoma with intramural and submucosal components who chose hysteroscopic resection instead of laparotomy and myomectomy before undergoing assisted reproduction.

\section{CASE REPORT}

The patient is a 45 -year-old white nulligravida with primary infertility for 10 years. During the past 4 years, she experienced three unsuccessful attempts at in vitro fertilization. Recently, a leiomyomatous uterus was discovered following an evaluation for intermenstrual bleeding. Her menstrual periods were irregular, with intervals of 45-60 days. The patient had elevated day 3 serum follicle-stimulating hormone (FSH) levels varying between 30 and $40 \mathrm{mIU} / \mathrm{mL}$. Transvaginal ultrasound revealed an enlarged uterus with an approximately $6 \times 6$ $\mathrm{cm}$ posterior-intramural leiomyoma with a submucosal component. Two other $3 \mathrm{~cm}$ leiomyomas were noted

Department of Obstetrics and Gynecology, University of Southern California School of Medicine, Los Angeles, California. 
laterally. The endometrial echo was noted to be contiguous with the largest tumor. An HSG demonstrated extension of the mass into the endometrial cavity (Fig. 1). Office hysteroscopy confirmed an approximately $3 \times$ $3 \times 2 \mathrm{~cm}$ intracavitary component. This represented approximately $40 \%$ of the tumor volume.

The patient was advised of the possible implications of a symptomatic submucosal leiomyoma. Concerns included the tumor's effect on implantation and the possibility for unscheduled bleeding, which might have a negative effect on implantation and early embryonic development. Furthermore, pregnancy might be complicated by large leiomyomas disrupting normal placentation and fetal development, as well as increasing the risks of both preterm labor and birth.

We discussed three treatment options: (1) proceeding without surgery, (2) undergoing laparotomy and myomectomy, and (3) undergoing hysteroscopic resection of the mass to restore a normal endometrial cavity. If abnormal bleeding could be ameliorated, the plan was to proceed with embryo transfer. The patient elected the last option and was scheduled for outpatient surgery.

Before undergoing surgery, the patient received gonadotropin-releasing hormone $(\mathrm{GnRH})$ agonist for 60 days in hopes of improving intrauterine visualization and shrinking the myoma. However, only minimal decrease in tumor size occurred, and intermittent bleeding persisted. Under general anesthesia, the patient underwent resection using a $27 \mathrm{~F}$ continuous flow gynecologic resectoscope with $1.5 \%$ glycine as a uterus-distending medium. The tumor was progressively shaved down to its base.

The patient did well postoperatively and was placed on a standard regimen of sequential oral micronized estradiol ( $2 \mathrm{mg}$ ) and medroxyprogesterone acetate $(5 \mathrm{mg})$ for 2 months, and normal withdrawal bleedings followed. Hysteroscopy was performed, and the cavity was normal. An endometrial biopsy while on hormone replacement therapy demonstrated a normal secretory response.

Oocyte donation was performed, and gametes were provided by a 30 -year-old fertile donor. Five preembryos were transferred transcervically, as described previously. ${ }^{2}$ Beta-hCG levels were increased 9 days after transfer, and ultrasound examination approximately 2 weeks later showed a single intrauterine gestational sac. The pregnancy was supported with estradiol and progesterone. The patient experienced no pelvic pain, and the leiomyoma maintained its size at approximately $4 \times 4 \mathrm{~cm}$ (Fig. 2). Hormones were discontinued by the 14th week of gestation, when serum levels of progesterone were above $50 \mathrm{ng} / \mathrm{mL}$.

The second and third trimesters were uncomplicated, and labor was induced at 40 weeks gestational age. The patient delivered a living, $3600 \mathrm{~g}$ female infant. The placenta was delivered without complications and found to be of normal size, weight, and contour. The patient did well and experienced minimal postpartum bleeding.

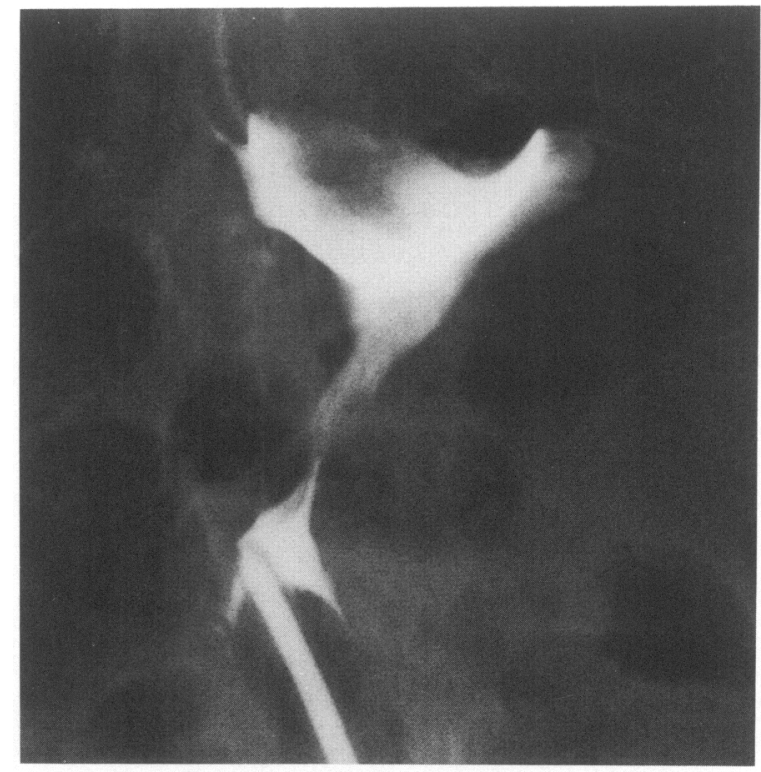

FIG. 1. Hysterosalpingogram performed before hysteroscopic resection showed the presence of a large intracavitary leiomyoma. 


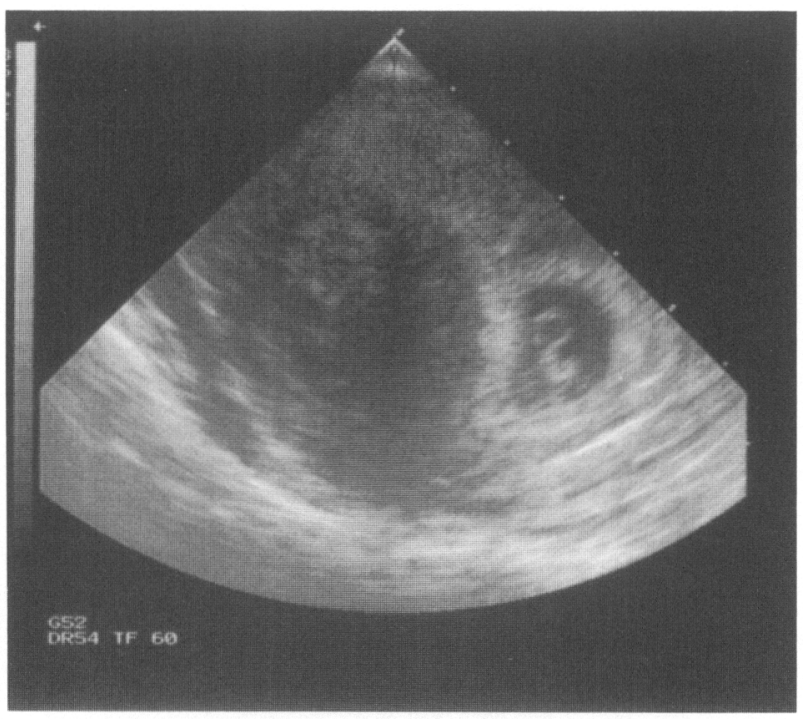

FIG. 2. The $4 \times 4 \mathrm{~cm}$ leiomyoma that remained unresected was visible on transvaginal ultrasound adjacent to the early pregnancy implantation site.

\section{DISCUSSION}

Oocyte donation has become increasingly popular for treating infertile women $40-50$ years of age. This method provides older infertile individuals the best opportunity for pregnancy. Recommendations have been to extend therapy only to patients with a normal endometrial cavity. This maximizes pregnancy potential by eliminating an adverse endometrial environment. In our experience, leiomyomas have been present in $25 \%$ of our oocyte donation patients. No decrease in overall embryo implantation or pregnancy rates was noted among asymptomatic patients with myomas if their uterine cavities were normal. However, women with leiomyomas in situ were significantly more likely to complain of pelvic pain during the first and second trimesters.

Hysteroscopic resection of leiomyomas has been advocated for treating symptomatic submucosal myomas. ${ }^{6}$ The role of hysteroscopic resection before pregnancy attempts remains undefined, although successful pregnancies have been reported. ${ }^{6}$ In patients experiencing abnormal uterine bleeding, it is probable that embryo transfer is adversely affected by bleeding. Endometrial shedding may increase the likelihood of washing out the free-floating embryo. Exposure to large numbers of white blood cells also might increase the probability that the zygote would be phagocytized.

This patient continued to bleed irregularly, and intracavitary extension of the lesion persisted despite GnRH agonist therapy. Therefore, surgery was recommended. Certainly, hysteroscopic resection has the advantage of being less morbid because there is no abdominal incision, blood loss is less, and it is an outpatient procedure. Understanding that the chance of success with embryo donation was approximately $35 \%$ per embryo transfer, the patient elected to take the most conservative approach.

This case represents the first report of a perimenopausal woman attempting pregnancy following a hysteroscopically resected submucosal leiomyoma and oocyte donation. It demonstrates the ability to reestablish a normal endometrial cavity capable of sustaining a pregnancy. However, more cases of hysteroscopic resection before embryo donation are needed to better define the value of this unique treatment regimen.

\section{REFERENCES}

1. Sauer MV, Paulson RJ, Lobo RA. Reversing the natural decline in human fertility. JAMA 1992;268:1275.

2. Navot D, Bergh PA, Williams MA, et al. Poor oocyte quality rather than implantation failure as a cause of age-related decline in female fertility. Lancet 1991;337:1375. 
3. Pantos K, Meimeti-Damianaki T, Vaxevanoglou T, Kapetanakis E. Oocyte donation in menopausal women aged over 40 years. Human Reprod 1993;8:488.

4. Society for Assisted Reproductive Technology. Assisted reproductive technology in the United States: 1991 results from assisted reproductive technology generated from the American Fertility Society Registry. Fertil Steril 1993;59:956.

5. Ary BA, Paulson RJ, Sauer MV. Pregnancy outcome in women undergoing oocyte donation with leiomyomata uteri or myomectomy. Surg Forum 1993;44:544.

6. Derman SG, Rehnstrom J, Neuwirth RS. The long-term effectiveness of hysteroscopic treatment of menorrhagia and leiomyomas. Obstet Gynecol 1991;77:591.

Address reprint requests to:

Mark V. Sauer, M.D.

Women's Hospital Room L-1013

1240 North Mission Road

Los Angeles, CA 90033 